\title{
The COVID-19 pandemic and colorectal cancer prevention: God tempers the wind to the shorn lamb
}

\author{
Referring to Zorzi M et al. p. 871-876 and Gralnek I et al. p. 891-898
}

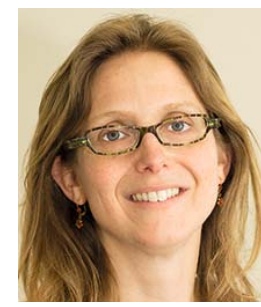

Maria Pellisé

\author{
Author \\ Maria Pellisé \\ Institution \\ Gastroenterology Department, Hospital Clínic de \\ Barcelona, Institut d'Investigacions Biomèdiques August Pi i \\ Sunyer (IDIBAPS), Centro de Investigación Biomédica en \\ Red de Enfermedades Hepáticas y Digestivas (CIBEREHD), \\ Barcelona, Spain \\ Bibliography \\ Endoscopy 2020; 52: 877-878 \\ DOI 10.1055/a-1243-1372 \\ ISSN 0013-726X \\ (C) 2020. Thieme. All rights reserved. \\ Georg Thieme Verlag KG, Rüdigerstraße 14, \\ 70469 Stuttgart, Germany \\ Corresponding author \\ Maria Pellisé, MD, Department of Gastroenterology, Hospital \\ Clínic, Villarroel 170, Barcelona, Catalonia 08036, Spain \\ Fax: +34-93-2279387 \\ mpellise@clinic.cat
}

The benefits of colorectal cancer (CRC) screening programs, in terms of cancer incidence and mortality, are indisputable. The medicalization of a large segment of the healthy and asymptomatic population is now well accepted as an investment to prevent future health harms and costs in the small proportion of individuals that could otherwise develop cancer. Indeed, CRC prevention has instigated a major shift in endoscopy practice. However, it has also generated an overwhelming workload for endoscopy units, which suffer from a limited and rigid capacity.

"... the findings confirm that there is no immediate need to rush a colonoscopy after a positive FIT result and that it is preferable to accept longer post-FOBT time lapses for colonoscopy scheduling than to pause population screening programs."

The COVID-19 pandemic represents an unprecedented health crisis. During the first wave, endoscopy activity has been limited to mostly emergency procedures. A recent study from the UK National Endoscopy Database, which is populated by automated real-time capture of endoscopy reports and contains over 2.5 million endoscopy records, revealed that the first weeks of the UK lockdown saw a reduction in endoscopy activity to $5 \%$ of normal, with activity only recovering to $20 \%$ of preCOVID-19 levels in the subsequent weeks [1]. After the lockdown, endoscopy services face two immense challenges: 1) the adaptation of endoscopy units and workflows to COVID-19 prevention measures, which include social distancing, enforced downtime, and additional cleaning between procedures, all of which reduce patient throughput; and, simultaneously, 2) the absorption of all postponed procedures into the already overloaded endoscopy agenda. Gastroenterology societies such as the European Society of Gastrointestinal Endoscopy have recommended that endoscopies be rescheduled according to indication, with therapeutic and diagnostic procedures in symptomatic patients scheduled before screening and surveillance procedures [2].

To avoid increasing the pressure on already saturated endoscopy departments, CRC population screening programs have been paused in most European countries. However, for patients with cancer, a delay in diagnosis and treatment has the real potential to increase the likelihood of diseases being found at more advanced stages, with some patients' tumors progressing from curable (with near-normal life expectancy) to non-curable 
(with limited life expectancy). Indeed, a recent modeling study indicated that even modest delays in cancer surgery of 3 to 6 months might significantly impact survival, particularly for stage 2 or 3 cancers [3]. As a matter of fact, the recent article from the UK National Endoscopy Database revealed a dramatic and worrying effect of the fall in endoscopy activity on cancer diagnosis, with up to $72 \%$ of expected CRCs not being detected [1]. Accordingly, major concern has arisen regarding the appropriateness of interruptions to such valuable programs.

In fecal occult blood test (FOBT)-based CRC screening programs, a delay between a FOBT-positive result and colonoscopy is associated with an increased risk of advanced CRC and mortality. A simulation model study estimated that a delay of 12 months could reduce the total years of life gained from screening by nearly $10 \%$ vs. a colonoscopy performed within 2 weeks after a positive FOBT [4]. Thus, it has been proposed that colonoscopy be delivered as soon as possible in patients with a likelihood of CRC and within 1 month in individuals with a positive FOBT. According to European guidelines, colonoscopy in FOBTpositive individuals should be scheduled within 31 days, and achievement of a $\leq 30$-day post-FOBT colonoscopy interval in $\geq$ $90 \%$ of cases is used as a quality indicator to audit the adequacy of population-based screening programs [5]. This highly demanding requisite is a cause of stress for endoscopy services, with post-FOBT colonoscopies having to compete with the other indications. This requirement may represent a currently insurmountable pressure and might lead to the interruption of CRC screening programs.

In this issue of Endoscopy, Zorzi et al. [6] report on the population-based screening program for CRC in the Veneto region of Italy and provide us with highly pertinent information on the current situation. The study included 123138 individuals who complied with an invitation to undergo post-fecal immunochemical test (FIT) colonoscopy. Despite the local recommendations, only $41 \%$ of patients had a time to colonoscopy< 30 days, and $58 \%$ had a time between 31 and 180 days. Although the detection rate for invasive CRC was stable for waiting times $<180$ days, a statistically significant excess was observed after a 270day cutoff (odds ratio 1.75, 95\% confidence interval 1.152.67). Moreover, the CRC stage was stable in relation to a waiting time $<270$ days.

The fact that a time to colonoscopy after FOBT up to 6 months was not associated with any increase in CRC prevalence or in CRC stage progression supports the overall safety of an extended time to colonoscopy after a positive FOBT. Despite the intrinsic limitations of the study by Zorzi et al. (a retrospective design with confounding factors that hamper causality deductions, a minority of patients with long delays, and an unknown CRC stage in one-third of cases), the findings are consistent with those of a large study from the USA [7] and another from Asia [8] that have evaluated CRC outcomes associated with variations in follow-up times. According to the report by Corley et al. [7], concerns regarding an increased risk of CRC and late-stage CRC arise about 10 months after a positive FIT result. Overall, the findings confirm that there is no immediate need to rush a colonoscopy after a positive FIT result and that it is preferable to accept longer postFOBT time lapses for colonoscopy scheduling than to pause pop- ulation screening programs. The present situation provides the ideal scenario to implement the prioritization of colonoscopies for FOBT-positive patients based on the previously identified risk factors for advanced neoplasia [9].

The results obtained by Zorzi et al. suggest that God does indeed temper the wind to the shorn lamb, with FOBT-positive patients able to safely wait up to 6 months before undergoing colonoscopy. Unexpectedly, the COVID-19 pandemic is affording us the opportunity to fine-tune our endoscopy activity by better adjusting it to the established indications and by tailoring waiting lists to procedures that evidently improve outcomes in terms of relevant diagnoses and life years saved.

\section{Competing interests}

M. Pellisé has received consultancy and speaker's fees from Norgine Iberia (2015-2019), a consultancy fee from GI Supply (2019), speaker's fees from Casen Recordati (2016-2019), Olympus (2018), and Jansen (2018), and research funding from Fujifilm Spain (2019), Fujifilm Europe (2020), and Casen Recordati (2020); her department has received loan material from Fujifilm Spain (from 2017 ongoing), a research grant from Olympus Europe (2005-2019), and loan material and a research grant from Fujifilm Europe (2020-2021); she is a Board member of ESGE and SEED; and receives a fee from Thieme as an Endoscopy Co-Editor.

\section{References}

[1] Rutter MD, Brookes M, Lee TJ et al. Impact of the COVID-19 pandemic on UK endoscopic activity and cancer detection: a National Endoscopy Database Analysis. Gut 2020: doi:10.1136/gutjnl-2020-322179

[2] Gralnek I, Hassan C, Beilenhoff U et al. ESGE and ESGENA Position Statement on gastrointestinal endoscopy and COVID-19: an update on guidance during the post-lockdown phase and selected results from a membership survey. Endoscopy 2020: 891-898

[3] Sud A, Jones ME, Broggio J et al. Collateral damage: the impact on outcomes from cancer surgery of the COVID-19 pandemic. Ann Oncol 2020; 31: 1065-1074

[4] Meester RGS, Zauber AG, Doubeni CA et al. Consequences of increasing time to colonoscopy examination after positive result from fecal colorectal cancer screening test. Clin Gastroenterol Hepatol 2016; 14: 1445-1451

[5] Moss S, Ancelle-Park R, Brenner $\mathrm{H}$ et al. European guidelines for quality assurance in colorectal cancer screening and diagnosis. First edition evaluation and interpretation of screening outcomes. Endoscopy 2012; 44: (Suppl. 03): SE49-SE64

[6] Zorzi M, Hassan C, Capodaglio G et al. Colonoscopy later than 270 days in a fecal immunochemical test-based population screening program is associated with higher prevalence of colorectal cancer. Endoscopy 2020; 52: 871-876

[7] Corley DA, Jensen CD, Quinn VP et al. Association between time to colonoscopy after a positive fecal test result and risk of colorectal cancer and cancer stage at Diagnosis. JAMA 2017; 317: 1631-1641

[8] Lee YC, Fann JCY, Chiang TH et al. Time to colonoscopy and risk of colorectal cancer in patients with positive results from fecal immunochemical tests. Clin Gastroenterol Hepatol 2019; 17: 1332-1340

[9] Auge JM, Pellise M, Escudero JM et al. Risk stratification for advanced colorectal neoplasia according to fecal hemoglobin concentration in a colorectal cancer screening program. Gastroenterology 2014; 147: 628-636 